\title{
Relación entre el tipo de funcionamiento familiar y la tendencia al riesgo suicida en adolescentes
}

\author{
Relationship between the type of family functioning and the tendency to suicidal \\ risk in adolescents
Relação entre o tipo de funcionamento familiar e a tendência ao risco de suicídio em adolescentes

\author{
Rauber Rodrigo Altuzarra Murillo \\ ro.altuzarra@gmail.com \\ https://orcid.org/0000-0002-2751-2298 \\ Universidad Autónoma “Juan Misael Saracho”, Tarija, Bolivia \\ Recibido: julio 2019 / Revisado: agosto 2019 / Aceptado: agosto 2019 / Publicado: septiembre 2019
}

RESUMEN

El objetivo de este estudio fue establecer la relación entre el tipo de funcionamiento familiar y la tendencia al riesgo suicida en adolescentes de nivel secundario que asisten al colegio José Manuel Belgrano, turno tarde, de la ciudad de Tarija. La metodología que se usó fue descriptivo-correlacional, con un enfoque cualitativo y diseño transversal. La población estuvo conformada por 403 estudiantes, y la muestra fue de 197 estudiantes. Las técnicas e instrumentos que se utilizaron para la recolección de datos, fueron inventario (test psicológico) para la tendencia suicida y funcionamiento familiar y los instrumentos fueron la escala de tendencia suicida de Poldinger y el funcionamiento familiar FACE III. Como resultado se obtuvo, la baja tendencia de suicidio en los adolescentes de dicha institución y se concluyó, que existe un alto predominio del tipo de funcionamiento familiar de rango medio en los estudiantes de nivel secundario.

Palabras clave:

Riesgo suicida; familia del suicida; familia; adolescente
ABSTRACT

RESUMO

The objective of this study was to establish the relationship between the type of family functioning and the tendency to suicidal risk in secondary-level adolescents who attend the José Manuel Belgrano school, afternoon shift, in the city of Tarija. The methodology used was descriptive-correlational, with a qualitative approach and crosssectional design. The population consisted of 403 students, and the sample was 197 students. The techniques and instruments used for data collection were inventory (psychological test) for suicidal tendency and family functioning, and the instruments were the Poldinger suicide tendency scale and FACE III family functioning. As a result, a low suicide tendency was obtained in adolescents from said institution and it was concluded that there is a high prevalence of the middle-range type of family functioning in secondary school students.

Key words:

Suicidal risk; suicide's family; family; teen
O objetivo deste estudo foi estabelecer a relação entre o tipo de funcionamento familiar e a tendência ao risco de suicídio em adolescentes do ensino médio que frequentam a escola José Manuel Belgrano, turno vespertino, na cidade de Tarija. A metodologia utilizada foi descritiva-correlacional, com abordagem qualitativa $e$ delineamento transversal, com população de 403 alunos e amostra de 197 alunos. As técnicas e instrumentos utilizados para a coleta de dados foram o inventário (teste psicológico) de tendência suicida e funcionamento familiar, e os instrumentos foram a escala de tendência ao suicídio de Poldinger e o funcionamento familiar FACE III. Como resultado, obteve-se baixa tendência ao suicídio nos adolescentes da referida instituição e concluiu-se que existe uma alta prevalência do tipo médio de funcionamento familiar em alunos do ensino médio.

Palavras-chave: Risco de suicídio; família do suicida; família; adolescente 


\section{INTRODUCCIÓN}

El funcionamiento familiar es la manera de interactuar de los miembros de la familia, se produce por las interacciones de la dimensión de cohesión (afecto) y adaptabilidad (autoridad). Se divide en tres tipos de familia; balanceadas, medias y extremas.

Por lo tanto, la cohesión familiar debe entenderse como, "el vínculo emocional o ligazón emocional que los miembros de la familia tienen entre sí" (Olson, 1985, citado por Ríos, 2014, p. 44). Incluye variables específicas tales como: ligazón emocional, fronteras, coaliciones, tiempo, espacio, amistades, toma de decisiones, intereses y formas de reacción. Al mismo tiempo, la adaptabilidad familiar se considera como: "la habilidad de un sistema familiar para cambiar su estructura de poder, las relaciones de roles y las reglas de las relaciones, en respuesta al estrés situacional y propia del desarrollo" (Olson, 1985, citado por Ríos, 2014, p.49), contiene las variables específicas: poder (capacidad de afirmación, control y disciplina), estilos de negociación, relaciones de roles y reglas de relación de la familia. (Olson, 1989)

Asimismo, es importante mencionar que, dentro de un sistema como la familia, la funcionalidad familiar se debe entender como:

La capacidad del sistema para enfrentar y superar cada una de las etapas del ciclo vital y las crisis por las que atraviesa. Esto significa que debe cumplir con las tareas encomendadas, que los hijos no presenten trastornos graves de conducta y que la pareja no esté en lucha constante. (Hernández, Cargill y Gutiérrez, 2011, p. 14).

En este sentido, es preciso entender a la vez que el suicidio;

es el acto por el que la persona se quita voluntariamente la vida, es una forma compleja de conducta que tiene causas biológicas, psicológicas y sociales, las cuales contribuyen a la muerte intencional para escapar de circunstancias dolorosas y como un acto de venganza contra otra persona. (Reyes Zubiría, 1999, Citado por Ríos 2014, p. 15).

De esta manera, es de vital importancia hablar entonces de la tendencia al riesgo suicida y entenderla como "aquella probabilidad que tiene un individuo de cometer un acto suicida a futuro, siendo un acto suicida cualquier acción intencionada con posibles consecuencias físicas en la que se pone en riesgo la vida" (Belfort, 2009, citado por Ríos 2014 p. 11).

Según Poldinger (1969), en todo suicida se produce una progresiva evolución hacia el suicidio, que él determina en tres estadios: en el primero, se considera la posibilidad, en el segundo, las tendencias constructivas y destructivas pugnan en el individuo y, finalmente, la tercera consiste en la decisión de suicidarse (p. 15).

A su vez, Pinillos (1981) indica que, la tendencia al suicidio es el resultado de un aprendizaje disfuncional, producto de una imitación de modelos personales o de condiciones socioculturales degradatorias de los mecanismos operantes de la conducta y de la propositividad natural de la misma" (p 166).

Aunque el suicidio se piensa, casi por definición, como un asunto meramente personal, al menos desde el siglo XIX la Organización Mundial de la Salud (OMS) ha reconocido el suicidio como un problema de salud pública, esto en vista de las tasas alarmantes que, en ciertas épocas y lugares, alcanzan las muertes por mano propia.

Por otra parte, el Ministerio de Salud, apoyado en un informe de la Fuerza Especial de Lucha Contra el Crimen (FELCC), da cuenta en un informe que en 2016 en La Paz hubo 1.139 (33\%) suicidios, 
le siguieron en forma descendente Cochabamba con 633 (18\%) suicidios, mientras que Santa Cruz reporta 572 (16\%); Potosí, 339 (10\%); Oruro, 308 (9\%); Chuquisaca, 285 (8\%); Tarija, 185 (5\%) y Pando registra 34 casos (1\%) (Beltrán, Gutiérrez, Medina, 2017).

El suicidio se puede producir a cualquier edad, y en 2015 fue la segunda causa principal de defunción en el grupo etario de 15 a 29 años en Tarija, Bolivia y todo el mundo. Notoriamente los suicidas son ahora mucho más jóvenes (Periódico El País, 2017).

En este sentido, el suicidio no es ajeno, ni extraño a la realidad tarijeña. Su impacto es desolador en el entorno familiar y social. Pero también es, un fenómeno que está latente. Sin embargo, queda claro que el abordaje sobre este problema ha sido muy escaso, en muchos casos no se toca, y es un estigma añadido al dolor y al sufrimiento de quienes se quedan aquí para llorarlo, vivirlo y guardar el secreto.

De esta forma, el estudio tiene por objetivo general, establecer la relación entre el tipo de funcionamiento familiar y la tendencia al riesgo suicida en adolescentes de nivel secundario que asisten al colegio José Manuel Belgrano, turno tarde, de la ciudad de Tarija.

Generando como aporte, brindar un entendimiento que procura generar reflexiones y discusiones sobre la importancia en atención al adolescente $\mathrm{y}$ en medidas preventivas en torno a la problemática, tanto dentro del colegio como en el hogar, orientado y dirigido hacia la población juvenil.

\section{Marco Teórico}

\section{Tendencia suicida}

El suicidio puede definirse como:

El acto por el que la persona se quita voluntariamente la vida, es una forma compleja de conducta que tiene causas biológicas, psicológicas y sociales las cuales contribuyen a la muerte intencional para escapar de circunstancias dolorosas y como un acto de venganza contra otra persona. (Reyes Zubiría, 1999, Citado por Ríos, 2014, p. 15).

El estudio de la tendencia al suicidio puede comprenderse como un espectro que abarca a las ideas y deseos suicidas (ideación suicida), las conductas suicidas sin resultado de muerte (intentos suicidas) y los suicidios consumados o completados (Durkheim, 1992, citado por Ríos, 2014).

La idea suicida puede ser tan detallada como un plan formulado, sin que el acto suicida finalice o puede ser toda la intención y tener éxito. Puede considerarse una expresión temprana de vulnerabilidad y abarca desde manifestaciones vagas de poco valor de la vida hasta propósitos autodestructivos más definidos. Las ideas suicidas se asocian a variables psicosociales, al medio familiar caótico las cuales pueden adoptar las siguientes formas de presentación: Idea suicida sin un método específico, con un método inespecífico o indeterminado en la que el individuo expone sus deseos de matarse, entre otras maneras.

Por lo tanto, la tendencia al riesgo suicida es entendida como "aquella probabilidad que tiene un individuo de cometer un acto suicida a futuro, siendo un acto suicida cualquier acción intencionada con posibles consecuencias físicas en la que se pone en riesgo la vida" (Belfort, 2009, citado por Ríos, 2014 p. 11).

Según Pinillos (1981):

La tendencia al suicidio es el resultado de un aprendizaje disfuncional, producto de una imitación de modelos personales o de condiciones socioculturales degradatorias de los mecanismos operantes de la conducta 
y de la propositividad natural de la misma. En una palabra, el suicida no nace, se hace. Solo que este hacerse del suicida representa una adquisición: nefasta, pero adquisición, esto es, un aprendizaje que se lleva a cabo en un medio sociocultural, por una persona que usa erróneamente de su libertad" (p. 166).

Consecutivo, existe un modelo neurobiológico que explica la intervención de factores biológicos y sociales del origen de la conducta suicida, el modelo de diátesis-estrés el cual considera que existen estresores intrínsecos y extrínsecos, se menciona a la depresión como mayor y como extrínsecos encontramos eventos vitales, dificultades financieras, pérdidas afectivas que son los precipitantes observables del acto suicida.

La diátesis o vulnerabilidad se caracteriza por una tendencia al pesimismo y desesperanza y se manifiesta como agresividad e impulsividad, estos pueden guiar al problema de comportamiento suicida (Tellez, y Forero, 2006).

\section{Fases del suicidio}

Poldinger (1969) sostiene tres estadios:

El primer estadio, el suicidio considerado como una posibilidad para resolver, mediante la destrucción de la propia vida, los problemas reales o aparentes. El impulso inicial puede partir de factores psicodinámicos como inhibición de agresiones y aislamiento social o de factores de sugestión, tales como: suicidios en la familia y en el ambiente próximo o noticia de prensa, literatura y "Films".

El segundo estadio, se caracteriza por la ambivalencia, indecisión y pugna íntima entre las tendencias constructivas y destructivas. Esta ambivalencia se manifiesta también mediante avisos de suicidio, que han de comprenderse como auténticas llamadas de socorro, pero que con frecuencia y a causa de prejuicios no son atendidas.
"Las personas que desean suicidarse no lo avisan de antemano y que aquellas, en cambio, que hablan de suicidarse, no lo hacen". Tal prejuicio ha costado ya la vida a innumerables seres humanos.

El tercer estadio, si el sujeto ha adoptado ya la decisión de quitarse lavida, hayque prestar atención sobre todo a los indicios indirectos. Habla en favor de una acentuación de la tendencia al suicidio, el hecho de que personas que, con anterioridad, se mostraban agitadamente angustiosas y deprimidas, tengan de pronto aspecto tranquilo y manifiesten una actitud aparentemente serena. Tal serenidad es sospechosa y equivale con frecuencia, a una "calma antes de la tormenta".

\section{La familia del suicida}

En este tipo de familias suele haber conflictos constantes, particularmente separaciones, y antecedentes patológicos especialmente de alcoholismo o alguna adicción en uno o ambos padres. En general, en estas familias existe una estructura alterada que se caracteriza por un sistema cerrado que inconscientemente prohíbe a sus miembros comunicarse entre sí y relacionarse estrechamente con el exterior. De manera que el joven se siente aislado dentro y fuera de casa, en donde tampoco la comunicación se da abiertamente.

En relación con el manejo afectivo, las familias de los adolescentes que tienden al suicidio, suelen mostrar un patrón agresivo de relación, con dificultades para expresar el enojo y la agresión, así como una depresión familiar generalizada. (Aserrí, 2004, pp. 25-26).

\section{Funcionalidad familiar}

El buen o mal funcionamiento de la familia es un factor determinante en la conservación de la salud o en la aparición de la enfermedad entre sus miembros. Con base en que la familia cumpla o deje de cumplir eficazmente sus funciones, se 
habla de familia funcional o disfuncional, en vez de referirse a ella como normal o patológica. La funcionalidad familiar es la capacidad del sistema para enfrentar y superar cada una de las etapas del ciclo vital y las crisis por las que atraviesa (Huerta, 1999, p. 37).

Así que, "la funcionalidad familiar cursa por cuatro etapas secuenciales: procesos de identidad, disposición al cambio, procesos de la información y estructuración de roles." (Velazco, R., y Chávez, V., 1994, citados por Munro, 2005).

\section{El papel de la familia en el intento suicida del adolescente}

En el proceso de consecución de una identidad positiva y estable como adulto, una de las tareas más importantes para el adolescente la constituye la adquisición de autonomía. El principal elemento de esta tarea consiste en una separación o distanciamiento gradual, en todos los sentidos, de la relación con sus padres. Para tal fin, la estrecha relación y dependencia de la familia inmediata empieza a ceder ante las relaciones más intensas con pares y adultos fuera de la familia; a medida que se experimentan nuevos desafíos, se requieren otros comportamientos y se adquieren diferentes responsabilidades. La base de las relaciones familiares, es el esfuerzo que realiza el adolescente por lograr su independencia, la cual conlleva cierto grado de conflicto. En este sentido, las relaciones familiares son una de las áreas críticas para el desarrollo de la personalidad del adolescente, porque involucran una relación particular entre él y su familia que puede rebasar sus recursos psicosociales. (Amezcua y Márquez, 1994).

A criterio del postulante debido a lo anteriormente expuesto, la familia puede convertirse en un factor determinante para el desarrollo psicológico y personal del joven. A través de la práctica de estilos o pautas educativas, de las relaciones e interacciones y presiones del sistema familiar, se crean situaciones que pueden estar directamente relacionadas con el intento suicida.

Características generales de la adolescencia que constituyen factores predisponentes de la conducta suicida.

En la adolescencia se incrementa la carga de presiones o responsabilidades individuales, que unido a la inexperiencia e inmadurez generan tropiezos que pueden traducirse en momentos de angustia, soledad y frustración, que propician factores de riesgo para cometer un acto o conducta suicida.

Estos adolescentes generalmente provienen de familias desestructuradas, con carencias en el aspecto económico, déficit sociales y culturales, alteraciones en las relaciones dentro y fuera del grupo familiar, o lo que se pudiera llamarse familias multiproblemas, o familias que por sus características intrafamiliares $\mathrm{y} / \mathrm{o}$ por el entorno en que viven pueden calificarse de alto riesgo, con pobreza educacional, y de exposición a situaciones familiares adversas (Cortés A., 2013).

Cabe destacar, que existen rasgos o atributos de la personalidad del adolescente que se convierten en factores de riesgo para cometer un acto suicida como lo es la poca tolerancia a la frustración, actitudes híper perfeccionistas, son críticos, rígidos intelectualmente, que no toleran el más mínimo fracaso, y a veces están convencidos de su propia maldad y no se sienten queridos. Las dificultades escolares son predictoras de ideaciones y conductas suicidas en esta etapa de la vida. Entre otros aspectos.

\section{MÉTODO}

La investigación se enmarcó bajo un estudio de tipo exploratorio, por dos motivos, la primera porque no se ha llegado a generar una investigación concreta acerca del tema a nivel regional, si bien existe al momento algunos acercamientos 
al tema, únicamente son guías e ideas generales relacionadas a los casos de suicidio y no así el empleo metodológico de una investigación que determine la relación de las variables principales. La segunda razón se debe a que la muestra que se tomó de estudiantes de nivel secundario alcanzó el $49 \%$ de la población seleccionada considerándose una muestra significativa.

Por tanto, la metodología que se usó fue descriptivo- correlacional, tomando en consideración la variable de la investigación; funcionamiento familiar y tendencia suicida, en estudiantes de nivel secundario del colegio José Manuel Belgrano de la ciudad de Tarija. Posterior, se realizó un análisis correlacional con los datos obtenidos.

A su vez, el estudio fue transversal. $Y$ se respaldó bajo el concepto de un estudio de tipo teórico, ya que la finalidad de esta investigación fue la de generar fundamentación teórica acerca de la relación que existe entre la tendencia suicida y el tipo de funcionamiento familiar.

Además, se desarrolló bajo un enfoque cualitativo, ya que el análisis estuvo bajo las directrices de un procedimiento y análisis estadístico, compuesto por el diagnóstico de tendencia suicida en adolescentes estudiantes de secundaria de la ciudad de Tarija y el tipo de funcionamiento familiar.

Por otro lado, la población que se usó para el desarrollo de la investigación estuvo conformada por los estudiantes de nivel secundario del colegio José Manuel Belgrano, turno tarde, de la ciudad de Tarija. De acuerdo a los datos proporcionados por el personal administrativo del colegio, estuvo compuesto por 193 hombres y 210 mujeres que comprenden una población total de 403 estudiantes, de edades comprendidas entre 14 a 18 años con un nivel de instrucción de 3ero a 6to de secundaria.
Mientras, se determinó una muestra significativa de 197 estudiantes, equivalente al $49 \%$ con relación a la población total, el muestreo fue obtenido mediante la fórmula de poblaciones finitas y la afijación proporcional de frecuencias, con un nivel de confianza del $95 \%$ y un margen de error del 5\%. La muestra fue seleccionada por el tipo de muestreo aleatorio estratificado.

Las técnicas e instrumentos que se utilizaron para la recolección de datos, fueron inventario (test psicológico) para la tendencia suicida y funcionamiento familiar y los instrumentos fueron la escala de tendencia suicida de Poldinger y el funcionamiento familiar FACE III.

La escala de tendencia suicida de Poldinger es un instrumento diseñado para evaluar la posibilidad de un suicidio en una o varias personas, se empleó un cuadernillo con 16 preguntas que permitió apreciar la suicidabilidad del evaluado, con un tiempo de administración 10 a 15 minutos. Para la confiabilidad de este instrumento, se empleó el coeficiente de Kuder-Richarson, el cual se interpretó como confiabilidad ALTA en las variables cuantitativas de la Escala de Tendencia Suicida. Es decir, el 0.7163 que es equivalente a $71.00 \%$ de confiabilidad que tiene el Instrumento de medición para determinar la tendencia de riesgo suicida.

La escala de funcionamiento familiar FACE III, es un instrumento diseñado para evaluar el tipo de funcionamiento familiar a través de las dos dimensiones mayores del modelo circumplejo de Olson como son cohesión y adaptabilidad familiar. El modelo permite que una persona clasifique a las familias en tres grupos generales, que corresponden a familias "balanceadas", "de rango medio" y "familias extremas". Este instrumento emplea un cuadernillo que consta de 20 ítems, donde la distribución interna y 10 ítems a la adaptabilidad. 


\section{RESULTADOS}

\section{Escala tendencia suicida de Poldinger}

Tabla 1. Tendencia al riesgo suicida en los estudiantes de nivel secundario que asisten al colegio José Manuel Belgrano.

\begin{tabular}{ccc}
\hline ALTERNATIVA & FRECUENCIA & $\%$ \\
\hline No riesgo suicida & 133 & $68 \%$ \\
Tendencia al riesgo suicida & 54 & $27 \%$ \\
Riesgo suicida & 10 & $5 \%$ \\
Alta probabilidad de riesgo suicida & 0 & $0 \%$ \\
Total & $\mathbf{1 9 7}$ & $\mathbf{1 0 0 \%}$ \\
\hline
\end{tabular}

De acuerdo con los resultados obtenidos a través de la escala de tendencia suicida de Poldinger para determinar la variable riesgo suicida en la población muestral evaluada, se pudo observar en la Tabla 1, que la mayor parte de los estudiantes de nivel secundario del colegio José Manuel Belgrano, turno tarde, de la ciudad de Tarija, se ve reflejado en un $68 \%$ con 133 estudiantes que no presentan una inclinación al riesgo suicida; en contraste se observa a un $27 \%$ con 54 estudiantes que reflejan una inclinación de Tendencia al Riesgo Suicida y por último, un $5 \%$ con 10 estudiantes se encuentra dentro del diagnóstico de riesgo suicida.

Con este último dato, se evidenció que, si bien está representado por un porcentaje muy bajo tiene una alta significatividad dentro del marco de estudio de esta investigación, debido a que aquellos estudiantes que se encuentran dentro del diagnóstico de riesgo suicida, tienen mayores probabilidades de cometer un acto suicida a futuro. Asimismo, existe la posibilidad de que estos estudiantes manifiesten o hayan manifestado una progresiva evolución hacia el suicidio, determinado en primer lugar por las ideaciones suicidas. Además, existe la posibilidad de que estos estudiantes manifiesten o hayan manifestado una progresiva evolución hacia el suicidio, determinado en primer lugar por las ideaciones suicidas.

De esta forma, se determinó a través de los hallazgos que el diagnóstico de no riesgo suicida es el que predomina en la población evaluada. Sin embargo, existe en menor grado una pequeña cantidad de personas que tienen una preocupante inclinación al riesgo suicida considerando la posibilidad de riesgo de intento suicida dentro de este pequeño grupo. Es importante comprender que:

La tendencia al suicidio es el resultado de un aprendizaje disfuncional, producto de una imitación de modelos personales o de condiciones socioculturales degradatorias de los mecanismos operantes de la conducta y de la positividad natural de la misma. En una palabra, el suicida no nace, se hace. Solo que este hacerse del suicida representa una adquisición: nefasta, pero adquisición, esto es, un aprendizaje que se lleva a cabo en un medio sociocultural, por una persona que usa erróneamente de su libertad." (Pinillos, 1981, p. 166). 
Tabla 2. Tendencia suicida según el sexo de los estudiantes de nivel secundario que asisten al colegio José Manuel Belgrano.

\begin{tabular}{lcccccc}
\hline \multicolumn{1}{c}{ ALTERNATIVAS } & \multicolumn{2}{c}{ SEXO } \\
& Femenino & \multicolumn{2}{c}{ Masculino } & \multicolumn{2}{c}{ Total } \\
& FRECUENCIA & $\%$ & FRECUENCIA & \% & FRECUENCIA & \% \\
\hline No riesgo suicida & 61 & 59 & 72 & 77 & 133 & 68 \\
Tendencia al riesgo suicida & 36 & 35 & 18 & 19 & 54 & 27 \\
Riesgo suicida & 6 & 6 & 4 & 4 & 10 & 5 \\
Alta probabilidad de riesgo suicida & 0 & 0 & 0 & 0 & 0 & 0 \\
Total & $\mathbf{1 0 3}$ & $\mathbf{1 0 0}$ & $\mathbf{9 4}$ & $\mathbf{1 0 0}$ & $\mathbf{1 9 7}$ & $\mathbf{1 0 0}$ \\
\hline
\end{tabular}

A través de los resultados obtenidos se pudo observar en la Tabla 2, la representación del cruce de variables entre la tendencia suicida según el sexo de la población muestral. En cuanto al sexo femenino se expresa que, 61 estudiantes que representan el $59 \%$ ubicándose dentro del diagnóstico de no riesgo suicida y a 6 estudiantes que representan el $6 \%$ que se encuentran dentro del diagnóstico de riesgo suicida; por otro lado, dentro del grupo de sexo masculino, se muestra que, 72 estudiantes representando el $77 \%$, se encuentran dentro del diagnóstico de no riesgo suicida y contrariamente a 4 estudiantes que representan al $4 \%$ dentro del diagnóstico de riesgo suicida.

Se pudo evidenciar que la proporción entre hombres y mujeres no presenta una diferencia significativa en cuanto a los resultados. Sin embargo, si se centra solo en el diagnóstico de tendencia al riesgo suicida se puede observar una diferencia ligeramente significativa donde existe un $35 \%$ de estudiantes del grupo femenino y en menor grado un $19 \%$ de estudiantes del grupo masculino.

Así que existe una diferencia ligeramente significativa entre hombre y mujeres, en su mayoría mujeres, quizá debido al hecho de que las mujeres suelen presentar tendencia suicida, pero no logran llevar a cabo un intento. En cambio, en los hombres al ser más impulsivos y tratar de mantener la fachada que el estándar masculino demanda, suelen no exteriorizar su sufrimiento llevándolo silenciosamente al acto suicida.

Tabla 3. Tendencia suicida según la edad de los estudiantes de nivel secundario que asisten al colegio José Manuel Belgrano.

\begin{tabular}{|c|c|c|c|c|c|c|c|c|c|c|c|c|}
\hline \multirow{3}{*}{ ALTERNATIVA } & \multicolumn{12}{|c|}{ EDAD } \\
\hline & \multicolumn{2}{|c|}{14 Años } & \multicolumn{2}{|c|}{15 Años } & \multicolumn{2}{|c|}{16 Años } & \multicolumn{2}{|c|}{17 Años } & \multicolumn{2}{|c|}{18 Años } & \multicolumn{2}{|c|}{ Total } \\
\hline & f & $\%$ & f & $\%$ & f & $\%$ & f & $\%$ & $\mathbf{f}$ & $\%$ & f & $\%$ \\
\hline No riesgo suicida & 30 & 71 & 43 & 75 & 33 & 61 & 24 & 65 & 3 & 43 & 133 & 68 \\
\hline Tendencia al riesgo suicida & 9 & 21 & 11 & 19 & 19 & 35 & 11 & 30 & 4 & 57 & 54 & 27 \\
\hline Riesgo suicida & 3 & 7 & 3 & 5 & 2 & 4 & 2 & 5 & 0 & 0 & 10 & 5 \\
\hline Alta probabilidad de riesgo suicida & 0 & 0 & 0 & 0 & 0 & 0 & 0 & 0 & 0 & 0 & 0 & 0 \\
\hline Total & 42 & 100 & 57 & 100 & 54 & 100 & 37 & 100 & 7 & 100 & 197 & 100 \\
\hline
\end{tabular}


En la Tabla 3 se devela el cruce de variables entre el diagnóstico de tendencia suicida según el grupo etario. Se aprecia en primer lugar, al grupo de 14 años de edad con 42 estudiantes donde 30 de ellos que corresponden al $71 \%$, se encuentran dentro del diagnóstico de No Riesgo suicida y, por otro lado, 3 estudiantes que representan el 7\% se encuentran dentro del diagnóstico de Riesgo suicida.

En segundo lugar, se muestra al grupo de edades de 15 años con 57 estudiantes donde 43 de ellos que responden al $75 \%$, se encuentran dentro del diagnóstico de No Riesgo suicida y, por otro lado, 3 estudiantes que representan el 5\% se encuentran dentro del diagnóstico de Riesgo suicida.

En tercer lugar, se muestra al grupo de edades de 16 años con 54 estudiantes donde 33 de ellos que responden al $61 \%$, se encuentran dentro del diagnóstico de No Riesgo suicida; contrariamente, 2 estudiantes que representan el $4 \%$ se encuentran dentro del diagnóstico de Riesgo suicida.

En cuarto lugar, se aprecia al grupo de edades de 17 años con 37 estudiantes donde 24 de ellos que responden al $65 \%$, se encuentran dentro del diagnóstico de No Riesgo suicida. Asimismo, 2 estudiantes que representan el $5 \%$ se encuentran dentro del diagnóstico de Riesgo suicida.

En quinto lugar, se divisa al grupo de edades de 18 años con una cantidad minoritaria de 7 estudiantes donde 3 de ellos que responden al $43 \%$, se encuentran dentro del diagnóstico de No Riesgo suicida y por otro lado 4 estudiantes que representan el $57 \%$ se encuentran dentro del diagnóstico de Tendencia al Riesgo suicida.

Por lo tanto, al respecto de estos resultados se pudo enfatizar que, de cada 7 adolescentes de 18 años 4 de ellos presentan tendencias al riesgo suicida, ya que, los resultados reflejaron dentro de este grupo etario un índice creciente dentro de este diagnóstico. Si bien los datos no apuntan a un riesgo suicida como tal y solo permanecen dentro de una tendencia, quizá se deba a que a esta edad los adolescentes en cierto modo van saliendo de aquella ambigüedad producida por la transición entre la adultez y la niñez. Sin embargo, esto no significa que estén libres del riesgo suicida, ya que esta condición depende de diferentes factores endógenos y exógenos al sujeto, cualquier persona a cualquier edad tiene probabilidades de presentar en algún momento de su vida tendencias al riesgo suicida.

\section{El funcionamiento familiar FACE III}

Tabla 4. Tipo de funcionamiento familiar de los estudiantes de nivel secundario que asisten al colegio José Manuel Belgrano.

\begin{tabular}{lcc}
\hline \multicolumn{1}{c}{ ALTERNATIVA } & FRECUENCIA & $\%$ \\
\hline Balanceada & 36 & 18 \\
Rango medio & 117 & 59 \\
Extremo & 44 & 22 \\
Total & $\mathbf{1 9 7}$ & $\mathbf{1 0 0}$ \\
\hline
\end{tabular}


De acuerdo a los resultados obtenidos a través de la aplicación de la escala de funcionamiento familiar FACE III para establecer el tipo de funcionamiento familiar global en los estudiantes de nivel secundario del colegio José Manuel Belgrano, turno tarde, de la ciudad de Tarija, se observa en la Tabla 4, el predominio de un 59\% con 117 estudiantes evaluados que corresponden a un tipo de funcionamiento familiar de Rango Medio; seguido de este dato encontramos un $22 \%$ con 44 estudiantes evaluados que corresponden a un tipo de funcionamiento familiar extremo, teniendo un funcionamiento familiar no funcional dentro de este último tipo de familia.

Por lo tanto, se evidenció que las familias de rango medio, son familias con un principio de liderazgo de los padres demasiado autoritarios, en algunos casos la disciplina es severa y predecible hacia los hijos y los padres son quienes toman la decisión designando responsabilidades y normas definidas; también las familias de este tipo están conformadas por miembros extremadamente individualistas, donde existe poca intimidad y lealtad familiar debido a la poca interacción de los miembros. A su vez, las familias extremas, son familias demasiado ineficaces en el control parental sin definición de roles claros o excesivamente rígidos; también dentro de este tipo de familias se encuentran aquellas con alta dependencia emocional donde el interés se focaliza únicamente en la familia y no se permiten tomar decisiones de forma independiente. En cualquiera de estos casos resultan problemáticos y pueden tener como consecuencia que el interés de los adolescentes se focalice fuera de la familia o cayendo en malas amistades, vicios, depresión o una progresiva evolución hacia el riesgo suicida.

En este sentido, las familias ubicadas en los extremos a pesar de ser consideradas como disfuncionales tienen particularidades especiales; como la capacidad de buscar soluciones a raíz de su caos, con lo que pueden aprender a generar cambios que podrían ser positivos y llegarían a un mejor funcionamiento, por lo que a nivel general de todas las familias es necesario cierto grado de conflicto para generar un entorno más funcional.

De acuerdo a los resultados obtenidos a través de la escala de funcionamiento familiar FACE III en relación a la dimensión de cohesión familiar, se muestra por medio de la Tabla 5 que en los estudiantes de nivel secundario del colegio José Manuel Belgrano, turno tarde, de la ciudad de Tarija, predomina la cohesión familiar de tipo desligado con un indicador de $49 \%$ con 97 estudiantes puntuados dentro de esta categoría. Por lo que son familias con una extrema separación emocional debido a la poca interacción entre sus miembros, donde predomina la separación personal y se toman decisiones de forma independiente.

Tabla 5. Tipo de cohesión familiar predominante en los estudiantes de nivel secundario que asisten al colegio José Manuel Belgrano.

\begin{tabular}{lcc}
\hline \multicolumn{1}{c}{ ALTERNATIVAS } & FRECUENCIA & \% \\
\hline Amalgamada & 10 & 5 \\
Conectada & 37 & 19 \\
Separada & 53 & 27 \\
Desligada & 97 & 49 \\
Total & $\mathbf{1 9 7}$ & $\mathbf{1 0 0}$ \\
\hline
\end{tabular}


Asimismo, se puede evidenciar en la Tabla 5, un índice del $5 \%$ con 10 estudiantes que corresponden a un tipo cohesión familiar amalgamado, el cual, son aquellas que extreman la cercanía emocional y tienen una fuerte dependencia y necesidad de lealtad hacia la familia. Probablemente este tipo de familias forme miembros inestables e inseguros para desenvolverse fuera del círculo familiar, acarreando serios problemas de autoestima o fracaso. Les resulta difícil salir de su zona de confort.

Por lo tanto, se estableció a través de los hallazgos que el tipo de cohesión familiar predominante en la población evaluada es del tipo Desligada, marcada por la ausencia de unión afectiva entre los miembros de la familia, ausencia de lealtad a la familia y alta independencia.

Por otro lado, se expresa en la Tabla 6, una ponderación del $4 \%$ con 8 estudiantes que corresponden a una adaptabilidad familiar de tipo rígida. Así que, se evidenció que, los miembros de una familia dentro de esta tipología, sufren de un exceso de responsabilidades y al verse en un ambiente de normas inflexibles sin la posibilidad de cambiar las reglas entran en crisis que desencadenan problemas a largo plazo como la separación de la familia, conflictos de identidad, autoestima, entre otros.

Tabla 6. Tipo de adaptabilidad familiar predominante de los estudiantes de nivel secundario que asisten al colegio José Manuel Belgrano.

\begin{tabular}{|c|c|c|}
\hline ALTERNATIVAS & FRECUENCIA & $\%$ \\
\hline Caótica & 90 & 46 \\
\hline Flexible & 47 & 24 \\
\hline Estructurada & 52 & 26 \\
\hline Rígida & 8 & 4 \\
\hline Total & 197 & 100 \\
\hline
\end{tabular}

Partiendo de los análisis obtenidos a partir de los resultados de la escala de funcionamiento familiar FACE III con relación a la dimensión de adaptabilidad familiar, se muestra a través de la Tabla 6, que en los estudiantes de nivel secundario del colegio José Manuel Belgrano, turno tarde, de la ciudad de Tarija, predomina la adaptabilidad familiar de tipo caótico con una ponderación de $46 \%$ con 90 estudiantes puntuados dentro de esta categoría.

Se pudo inferir que estas características en la familia de tipo caótica se dan debido a la falta de responsabilidad de los padres de familia y la falta de límites familiares como la definición de roles claros y congruentes; al mismo tiempo supone una condición familiar donde no existen normas y probablemente las reglas cambian constantemente.

En este sentido, se enfatizó que la adaptación familiar de tipo caótico predomina en los adolescentes que fueron evaluados. Lo que evidenció alteraciones en la dinámica familiar y la posibilidad de que, en las familias de los adolescentes, los roles en el hogar no sean claros, no se cumplan de forma efectiva, que los padres no tengan autoridad ni liderazgo en su dinámica familiar, causando frecuentes cambios en las reglas, y que las decisiones parentales sean impulsivas; situación que se puede presentar debido a la ausencia de uno o ambos padres en el hogar por motivos laborales o fallecimiento. 


\section{CONCLUSIONES}

Posterior a los análisis de los resultados y dando cumplimiento al objetivo general de la investigación se pudo concluir que existe un predominio del diagnóstico de no riesgo suicida. Sin embargo, una cuarta parte de estudiantes presenta una inclinación de tendencia al riesgo suicida, seguido de un pequeño grupo de estudiantes dentro del diagnóstico de riesgo suicida, este último grupo representó al 5\% con 10 estudiantes. Si bien este porcentaje es bajo, recae sobre una alta significatividad desde el punto de vista cualitativo, considerando que este grupo de estudiantes tiene mayores probabilidades de cometer un acto suicida a futuro. De este modo se rechaza que, los estudiantes de nivel secundario que asisten al colegio J. M. Belgrano de la ciudad de Tarija presentan un alto índice de tendencia al riesgo suicida.

Con relación al tipo de familia, se pudo establecer que el predominante es de rango medio, entendiendo que el tipo de funcionamiento de estas familias no es del todo saludable, debido a que son propensas a la disfuncionalidad caracterizándose por un liderazgo limitado, ausencia de claridad en sus funciones, cumplimiento demasiado estricto de reglas y ausencia de límites; en este tipo de funcionamiento se encuentran las familias moderadamente funcionales. De acuerdo a estos resultados se afirma que existe un alto predominio del tipo de funcionamiento familiar de rango medio en los estudiantes de nivel secundario que asisten al colegio J. M. Belgrano de la ciudad de Tarija.

\section{REFERENCIAS}

Amezcua, R., y Márquez, E. (1994) El suicidio en los adolescentes, Obtenida el 20 de octubre de 2017, de http://www.scielo.org.mx/ scielo.php?script=sci_arttext\&pid=S003636342005000100001
Aserrí, L. (2004). Prevención de suicidio y conductas autodestructivas en jóvenes. http://www. binasss.sa.cr/adolescencia/aserri1.pdf

Beltrán, J.; Gutiérrez, N., y Medina, I. (2017). Suicidio en adolescentes, un problema pendiente en Bolivia. Rev. Página Siete. https:// www.paginasiete.bo/gente/2017/11/16/ suicidio-adolescentes- problema-pendientebolivia-159648.html

Cortés, A. (2013). Conducta suicida adolescencia y riesgo. Instituto Nacional de Higiene, Epidemiología y Microbiología (INHEM): La Habana

El suicidio, un tabú. (2017). Obtenido el 13 de julio de 2017, página web del periódico El País. http://elpaisonline.com/index.php/sociales-2/ item/255438-el- suicidio-un-tabu

Hernández, L.; Cargill, N., y Gutiérrez, G. (2011). Funcionalidad familiar y conducta de riesgo en estudiantes de nivel medio superior. Salud en Tabasco, vol. 18, Estado de Tabasco Villahermosa, México

Huerta, J. (1999). Estudio de salud familiar en programa de actualización continua en medicina familia 1. México

Munro, S. (2005). Evaluación de la autoestima y su relación con la funcionalidad familiar en los médicos de las unidades de medicina familiar. Colima, Colima

Olson, D. (1985). Family adaptability and cohesion evaluation scales (FACES III). Minnesota: Ed. Family Social Science

Olson, D.; Russell, C., y Sprenkle, D. (1989). Cirumplex model: Systemic Assessment and Treatment of Families. New York: Haworth Press

Pinillos, J. (1981). Tres lecciones sobre psicología del suicidio. Estudios Penales y Criminológicos, vol. IV. Cursos e Congresos $n^{\circ} 18$. Servizo de Publicacións da Universidade de Santiago de Compostela. ISBN 84-7191-243-0, pp. 138-168 Poldinger, W. (1969). La tendencia al suicidio. Madrid: Ediciones Morata

Ríos, J. (2014). Funcionamiento familiar y riesgo suicida en estudiantes que acuden a clínica universitaria en la ciudad de Huancayo. Lima, Perú

Tellez, J., y Forero J. (2006). Suicidio: Neurobiología, factores de riesgo y prevención. Bogotá: Nuevo Milenio Editores 